\title{
Off-Equilibrium Dynamics of a 4D Spin Glass with Asymmetric Couplings
}

\author{
E. Marinari ${ }^{1}$ and D. A. Stariolo ${ }^{2}$ \\ 1- Dipartimento di Fisica and INFN, Università di Cagliari \\ Via Ospedale 72, 09100 Cagliari (Italy) \\ 2- Departamento de Física, Universidade Federal de Viçosa * \\ 36570-000 Viçosa, MG (Brazil)
}

\begin{abstract}
We study the off-equilibrium dynamics of the Edwards-Anderson spin glass in four dimensions under the influence of a non-hamiltonian perturbation (i.e. with asymmetric couplings). We find that for small asymmetry the model behaves like the hamiltonian model, while for large asymmetry the behavior of the model can be well described by an interrupted aging scenario. The autocorrelation function $C\left(t_{w}+\tau, t_{w}\right)$ scales as $\tau / t_{w}^{\beta}$, with $\beta$ a function of the asymmetry. For very long waiting times the previous regime crosses over to a time translational invariant regime with stretched exponential relaxation. The model does not show signs of reaching a time translational invariant regime for weak asymmetry, but in the aging regime the exponent $\beta$ is always different from one, showing a non trivial aging scenario also valid in the Hamiltonian model.
\end{abstract}

\section{Introduction}

The interest toward the study of spin models with asymmetric couplings began in the last decade in connection with neural network models. Here it is known that the synapses (the connections between neurons) are not symmetrical. In order to analyze an asymmetric network one is forced to go beyond equilibrium statistical mechanics, since the system cannot be described by a Hamiltonian: a purely dynamical analysis is, in general, a very complex task. The first big efforts in this direction were made in [1]. Within the framework of a dynamical mean field theory, first developed in the case of spin glasses [2], it was possible to study analytically a very simplified asymmetric model with a spherical constraint. The authors reached the very interesting conclusion that the spin glass phase is suppressed by an arbitrary small amount of asymmetry. This property was considered relevant in the neural network community, as in this context the spin glass phase corresponds to spurious

*e-mail: stariolo@mail.ufv.br 
states that deteriorate the memory properties of the network. On the other side, without a spin glass phase, the dynamical properties of asymmetric networks seemed to be trivial and the interest in those systems diminished. In the last years we have improved our understanding of the dynamics of glassy systems, in particular of the so-called aging phenomena (see for example [3]). It is now clear that the mean field dynamics of spin glasses has to be modified in order to account for the fact that spin glasses are out of equilibrium. That implies, for example, that the dynamics is not time translational invariant and as a consequence the classic fluctuation-dissipation theorem is not valid anymore.

With regard to asymmetric systems, one of the main questions is to what extent the asymmetry affects the aging scenario. The conclusions of [四] seemed to indicate that a system with asymmetry will not age (there is no a spin glass like behavior).

The work of the following years has mainly concerned extreme situations like the fully asymmetric model and the $T=0$ case (see for example [4] and references therein). Recently the dynamical and static properties of the Sherrington-Kirkpatrick model with asymmetric couplings have been studied in [5]. It has been found that the off-equilibrium dynamics of the model is definitely not the one predicted by the spherical approximation and an aging scenario indistinguishable from the one of the Hamiltonian mean field model is clearly present for a wide range of small asymmetries.

In an other recent paper [6], from an analysis of the off-equilibrium dynamics of an asymmetric $p$-spin spherical model, the authors find that, although for random initial conditions aging is destroyed, by carefully tuning the initial conditions one is able to find regions where the system ages forever, never reaching equilibrium.

These findings leave open the discussion about the nature of the dynamics of asymmetric networks. Since almost all the known analytic properties of asymmetric networks are based on mean field spherical models, one has to be careful in extrapolating the conclusions to other, more realistic, models.

Here we discuss the issue of whether the overall behavior seen in mean field models survives in systems with finite range interactions. We have done computer simulations of the four dimensional Edwards-Anderson model with asymmetric interactions. From a careful analysis of the off-equilibrium dynamics we are able to present a clear picture of what happens in finite dimensions. The choice of $D=4$ is because the spin glass transition in the symmetric EA model is clearly established while the more realistic case of $D=3$ is still a matter of (little) debate [7].

Our simulations of the $D=4$ model clearly show that the autocorrelation functions behave according to a typical aging pattern】, for several values of the asymmetry. For strong asymmetries aging is interrupted and time translational invariance (TTI) is restored after a transient time scale. The scaling in the aging region is not a simple one. We notice that when assuming the form $C\left(t_{w}+\tau, t_{w}\right)=\tilde{C}\left(\tau / t_{w}^{\beta}\right)$, with the exponent $\beta$ depending on the degree of asymmetry (and probably on the temperature), one obtains very good fits. For long enough waiting times in the case of strong asymmetry the dynamics becomes stationary (TTI) and the decay can be well fitted by a stretched exponential. For weak asymmetry the systems cannot reach the stationary regimes for the time scales simulated but the aging scenario observed is clearly not a simple one (i.e. with scaling $\tau / t_{w}$ ): in

\footnotetext{
${ }^{1}$ It is clear now that aging for the SK model is not simple aging, but a more complex behavior [8]. This is what we mean here and in the rest of this note by typical aging.
} 
this case we find a behavior that cannot be distinguished from the one of the Hamiltonian model [5].

\section{The Model}

Our model system is defined by the Edwards-Anderson spin glass Hamiltonian:

$$
H=-\sum_{<i, j>}^{N} s_{i} J_{i j} s_{j},
$$

where $\left\{s_{i}= \pm 1, i=1 \ldots N\right\}$ are $N$ Ising spins and $\langle i, j\rangle$ denotes a sum over nearest neighbors. The couplings $J_{i j}$ are chosen as in [5]. They are a weighted sum of a symmetric and a completely asymmetric part:

$$
J_{i j}=\frac{1}{\sqrt{1-2 \epsilon+2 \epsilon^{2}}}\left[(1-\epsilon) J_{i j}^{(S)}+\epsilon J_{i j}^{(N S)}\right] .
$$

The symmetric part of the interaction is given by the symmetric couplings $J_{i j}^{(S)}=J_{j i}^{(S)}= \pm 1$ with probability 0.5 . The coupling $J_{i j}^{(N S)}$ gives the non-symmetric part and it is drawn independently of $J_{j i}^{(N S)}$. Finally, $\epsilon$ measures the strength of the non-symmetric part.

For the simulations we have chosen a standard heat bath dynamics and, starting from a random initial condition, we let the system evolve at temperature $T$ during a waiting time $t_{w}$. Then we measure the autocorrelation function at times $t=t_{w}+\tau$ :

$$
C\left(t_{w}+\tau, t_{w}\right)=\frac{1}{N} \sum_{i=1}^{N} \overline{\left\langle s_{i}\left(t_{w}\right) s_{i}\left(t_{w}+\tau\right)\right\rangle} .
$$

As usual, the overline means a disorder average while the brackets mean a thermal average. We measure the autocorrelation for several values of the waiting time, of the form times $2^{n}$.

In the following we will describe the results obtained for a lattice of linear dimension $L=7$. We have done also simulations for $L=5$ and $L=6$ and confirmed that finite size effects do not play an important role in our conclusions. Remembering that the critical temperature of the symmetric model with $\pm J$ interactions is approximately $T_{c} \approx 2$, we have done the simulations at a fixed temperature $T=0.5$.

Before presenting our results let us briefly describe, for reference purpose, the behavior found in the model with $\epsilon=0$, i.e. the Hamiltonian symmetric case, which dynamic properties (for a Gaussian distribution of the couplings) were studied in [9]. The authors found that the $4 D$ spin glass in the low temperature phase presents a typical aging behavior that can be well characterized by the following scaling of the autocorrelation:

$$
C\left(t_{w}+\tau, t_{w}\right)=\tau^{-x(T)} \tilde{C}\left(\tau / t_{w}\right)
$$

with a scaling function

$$
\tilde{C}(z)= \begin{cases}\text { constant } & \text { for } z \rightarrow 0 \\ z^{x(T)-\lambda(T)} & \text { for } z \rightarrow \infty\end{cases}
$$


In the "quasi-equilibrium" regime where $z \rightarrow 0$ one can write that $\lim _{t \rightarrow \infty} \lim _{t_{w} \rightarrow \infty} C\left(t, t_{w}\right)=$ $q_{E A}$, where $q_{E A}$ is the Edwards-Anderson order parameter. In the other regime there is a faster decay of the autocorrelation toward zero, i.e. $C \approx t^{-\lambda}$ with $\lambda(T) \gg x(T) \forall T$.

In the following we will discuss how this picture is modified when an asymmetric perturbation is introduced in the system.

\section{Results}

In figure (1) we show a log-log plot of the autocorrelation function $C\left(t_{w}+\tau, t_{w}\right)$ versus $\tau$, for several values of the asymmetry $\epsilon=0.1,0.2,0.3$ and 0.4 . For each case we plot the curves for $t_{w}=2^{n}$ with $n=2,5,8,11,14 \ldots$ As the asymmetry grows we can readily see a departure from the two regimes scenario that is valid in the symmetric model. For the cases with larger asymmetry the scaling of the symmetric model is no longer valid and, at least for $\epsilon>0.2$, an interrupted aging scenario is evident: for large $t_{w}$ correlation functions do not change anymore. The situation is more subtle for smaller values of $\epsilon$ and, for the time scales reached in this simulations, this effect is not detectable: here we find that on our time scales the typical aging scenario persists. The overall picture of the high asymmetry case is reminiscent of the one found for the $2 D$ spin glass [10], where there is no spin glass phase but at low temperatures one still observes interrupted aging scenario.

In order to make quantitative predictions we have run longer simulations for the model with $\epsilon=0.3$ going up in time to $t_{w}=2^{19}$. We have then tried a data collapse in the following form: take the data for a particular $t_{w}$ that we have chosen for practical purposes to be the largest one $\left(2^{19}\right)$. Then for each other value of $t_{w}$ we have tried a transformation of the form $C(x) \rightarrow a C(b x)$, adjusting the parameters $a$ and $b$ to make the curves for the two waiting times collapse. We plot $\log b$ versus $\log t_{w}$ in figure (2). The result is very interesting. First we note an intermediate region, for $2^{9} \leq t_{w} \leq 2^{12}$, where the function is linear $b \propto t_{w}^{\beta}$. This suggests that in this region the autocorrelation scales as:

$$
C\left(t_{w}+\tau, t_{w}\right) \sim \tilde{C}\left(\frac{\tau}{t_{w}^{\beta}}\right)
$$

Doing a linear fit of these four points we obtain for the exponent $\beta=0.34$. For waiting times greater than $t_{w}=2^{13}$ the system enters another regime with a nearly constant value of $b \approx 1$. This means that for $t_{w} \approx 2^{15}$ the dynamics changes qualitatively and enter a time translational invariant regime, thus the aging is interrupted. So there is a typical

relaxation time $t_{w}^{M A X}$ which signals the onset of a stationary regime. In order to test if this stationary regime presents a simple exponential relaxation we have done a log-log plot of the function $-\tau / \log \left(C\left(t_{w}+\tau, t_{w}\right)\right)$ versus $\tau$ for $t_{w}=2^{9}, 2^{11} \ldots 2^{19}$ as shown in figure (3)). As expected the curves saturate in a limit curve for $t_{w} \geq 2^{15}$ but the relaxation turns out not to be a simple exponential. If this were the case the limiting curve would be constant, with zero slope. The straight lines with finite slope observed are evidence of a stretched exponential relaxation,

$$
C(\tau)=e^{\tau^{\alpha} / t^{*}}
$$

where $t^{*}$ is the characteristic time of the relaxation. 

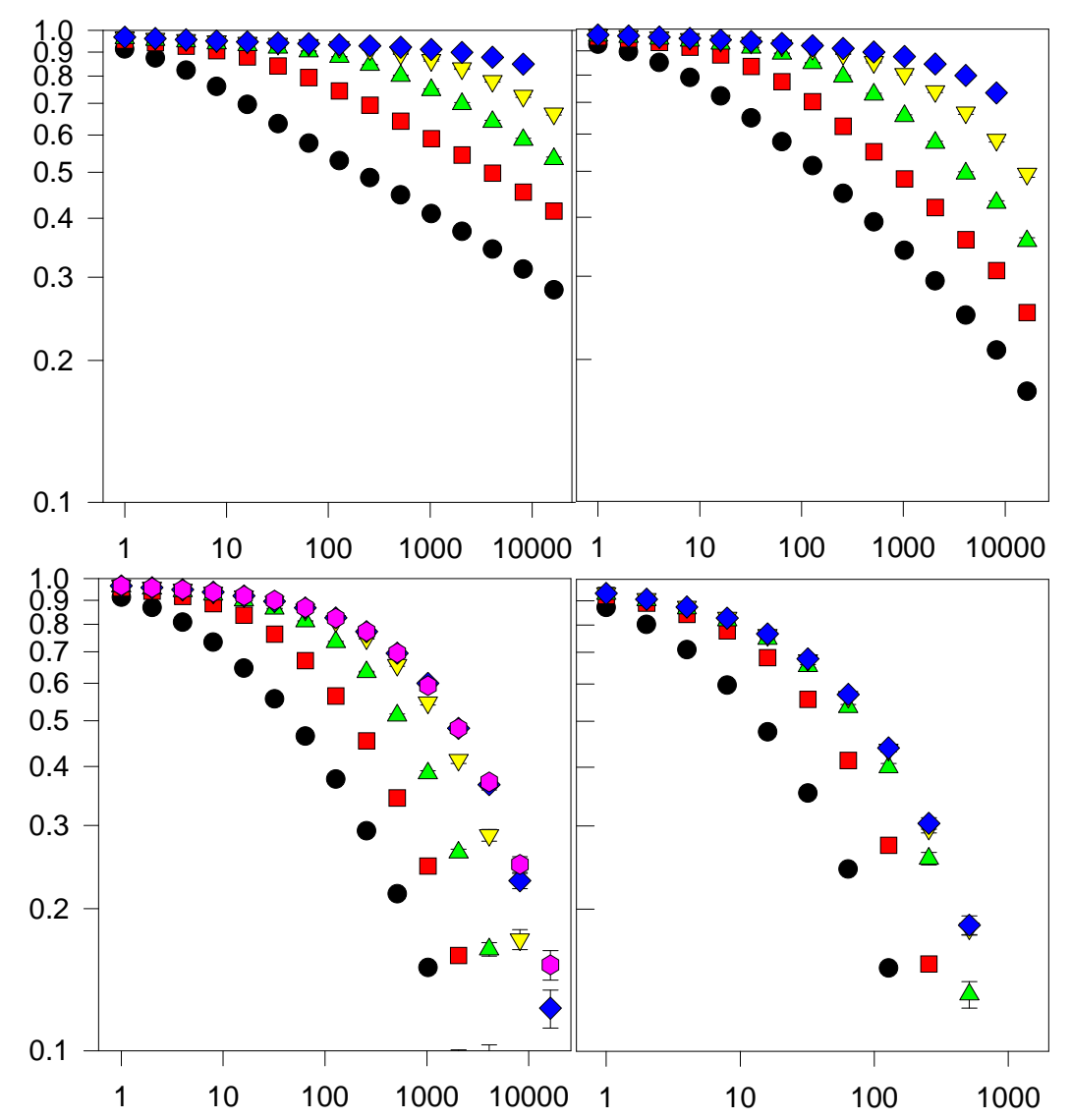

Figure 1: Autocorrelation functions for $T=0.5$, asymmetries $\epsilon=0.1,0.2,0.3$ and 0.4 and different waiting times (see text). 


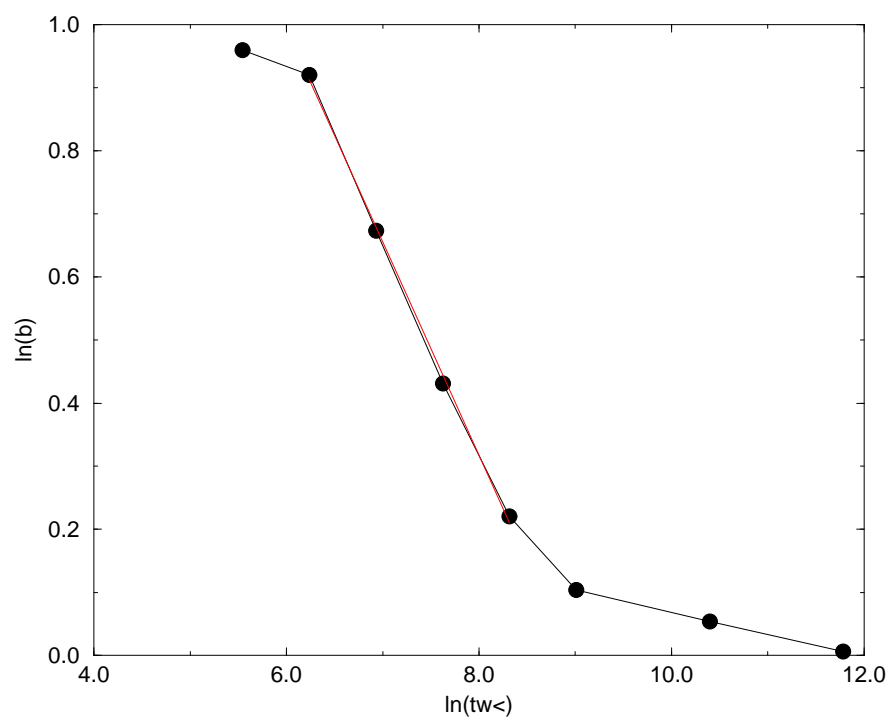

Figure 2: Data collapse of the autocorrelation for $\epsilon=0.3$ (see text).

In figure (4) we show a scaling plot of the autocorrelation function in the aging region. This figure has to be compared with Fig.(5) of Ref. [9] for the symmetric model. It is clear that now there are not two sharply defined time regimes and the scaling with $\tau / t_{w}^{\beta}$ is very good in the whole interval of the figure.

For growing asymmetry $t_{w}^{M A X}$ is smaller, as expected. The aging dynamics is interrupted earlier and a stationary dynamics dominates the scene. For $\epsilon=0.4$ the proposed scaling of the autocorrelation still works well with an exponent $\beta=0.32$ for $2^{5} \leq t_{w} \leq 2^{9}$ as can be seen in figure (5). For $t_{w} \geq 2^{10}$ a stationary regime clearly sets in with a characteristic stretched exponential relaxation. The scaling in the aging region is shown in figure (而).

Already for $\epsilon=0.5$ the complete dynamics is not slow any more, and the aging regime is completely suppressed. One could guess that also the stretched exponential relaxation would change to a simpler exponential one at some $\epsilon<1$ but our results up to $\epsilon=0.7$ still show the stretched exponential behavior.

For small values of $\epsilon=0.1$ and $\epsilon=0.2$ the dynamics resembles very much that of the symmetric model, at least up to the time scales we were able to simulate. For $\epsilon=0.1$ the proposed scaling for the aging regime works well in the whole time window from $2^{9} \leq t_{w} \leq 2^{14}$ with an exponent $\beta=0.90$, as one can see from the linear fit in figure (0). In figure (8) we show that in this case there are two well defined regimes with a behavior very similar to that of the symmetric case (see Fig.(5) of [9]). Our scaling works very well in the long time regime when $\tau \geq t_{w}^{\beta}$. The other corresponds to the quasi-equilibrium one where the autocorrelation depends only on the time difference $\tau$. 


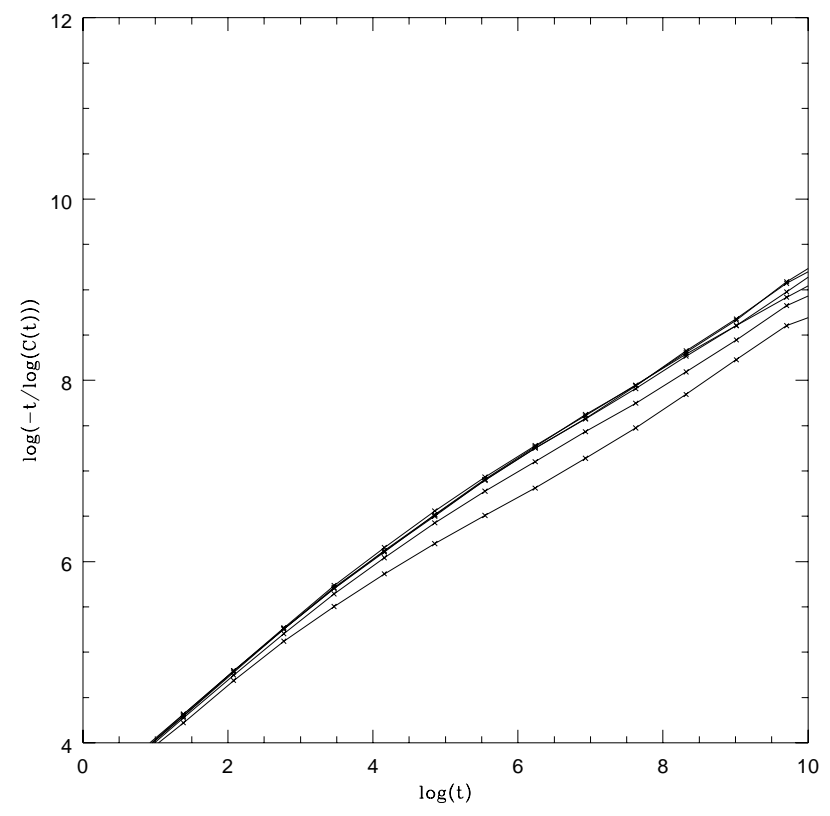

Figure 3: Stretched exponential behaviour of the autocorrelations for $\epsilon=0.3$ and waiting times $t_{w}=2^{9}, 2^{11} \ldots 2^{19}$ from bottom to top. 


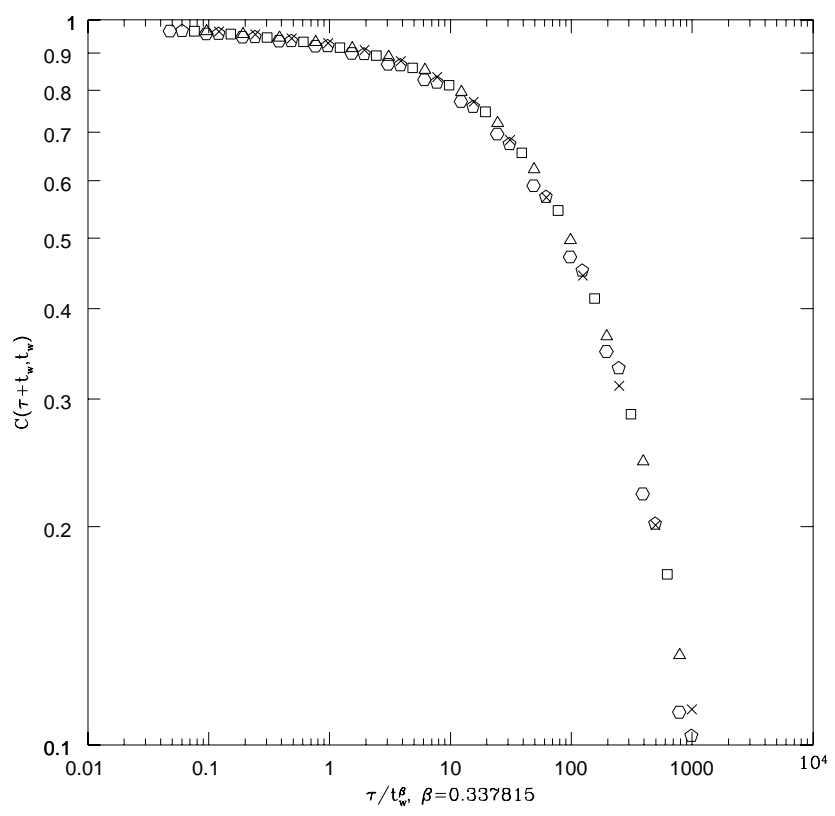

Figure 4: Scaling plot of the autocorrelation function in the aging regime for $\epsilon=0.3$.

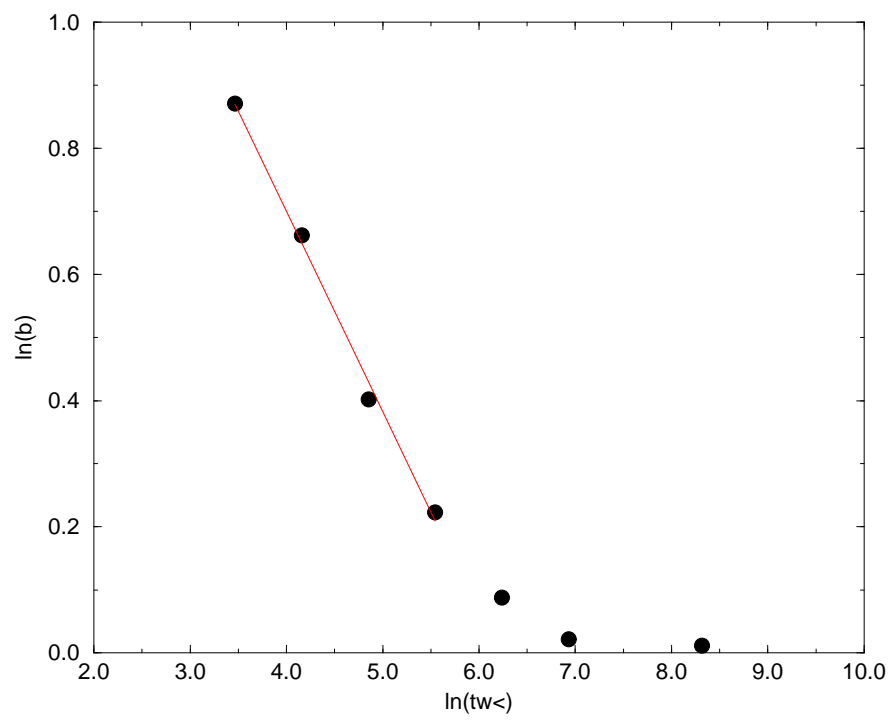

Figure 5: Data collapse of the autocorrelation for $\epsilon=0.4$ (see text). 


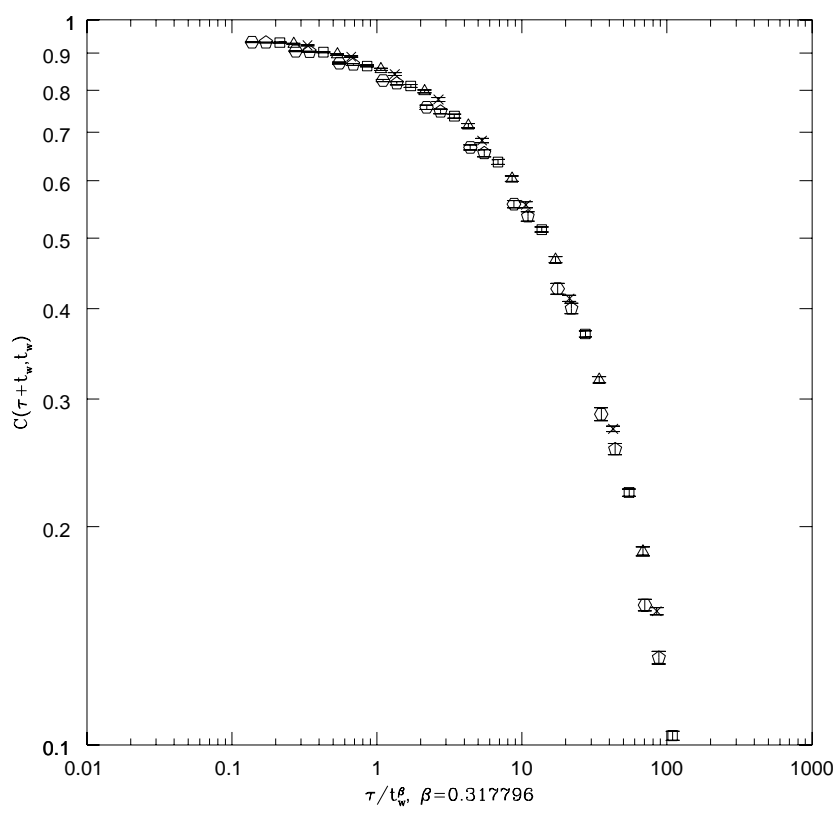

Figure 6: Scaling of the autocorrelation in the aging regime for $\epsilon=0.4$.

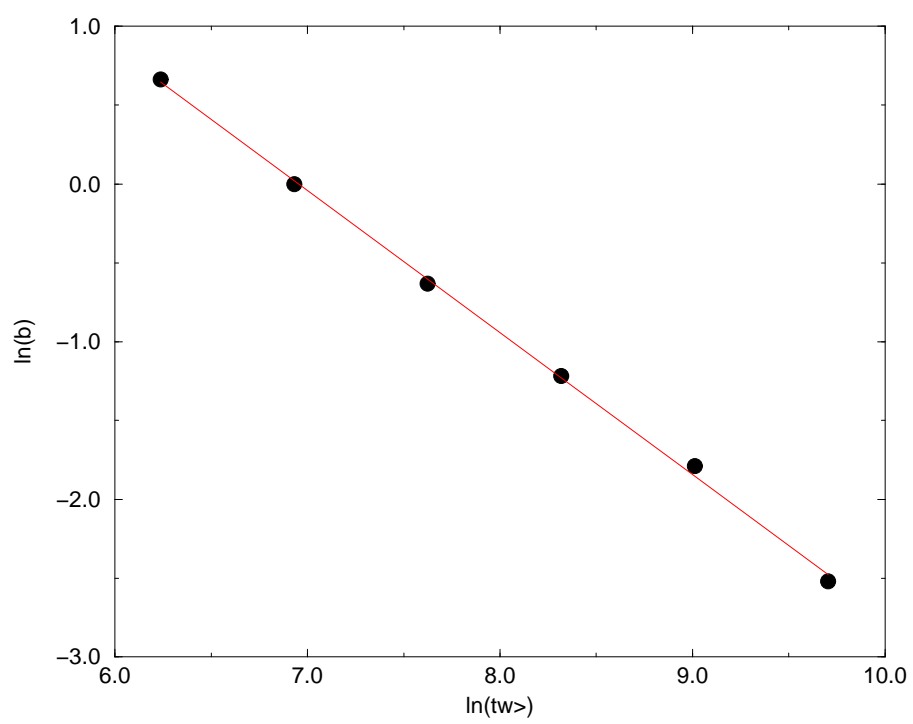

Figure 7: Data collapse of the autocorrelation for $\epsilon=0.1$ (see text). 


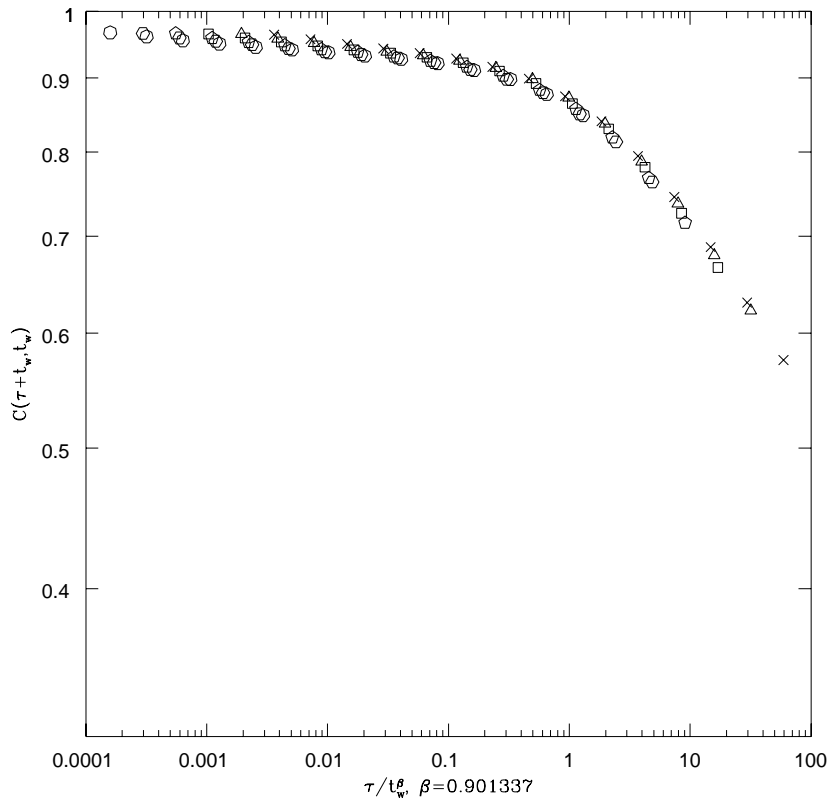

Figure 8: Scaling of the autocorrelation for $\epsilon=0.1$ and $t_{w}=2^{9} \ldots 2^{14}$.

For $\epsilon=0.2$ the division in two well defined regimes begins to break down and the crossover region is larger (see figure (94)). Nevertheless the aging scaling works well in the whole time window explored and a good data collapse is obtained with an exponent $\beta=0.72$. In our simulations, for this two values of $\epsilon=0.1$ and 0.2 , it is very difficult to see a maximum waiting time which signals the interruption of the aging. Here longer runs would be needed.

\section{Conclusions}

Finite dimensional disordered systems with quenched asymmetric couplings behave very much like the Hamiltonian systems if the asymmetry is small. Here we have shown that we observe indeed two kinds of behavior: for small asymmetry a typical aging, and for large asymmetry an interrupted aging. We have been able to qualify in good detail the large asymmetry phase, where reasonable correlation times make possible a good description of the dynamical behavior. We have suggested that a stretched exponential behavior can be here explicative of many observed features. A more detailed analysis is needed for a full understanding of the small asymmetry phase, where it is very difficult to observe any difference from the pure case. 


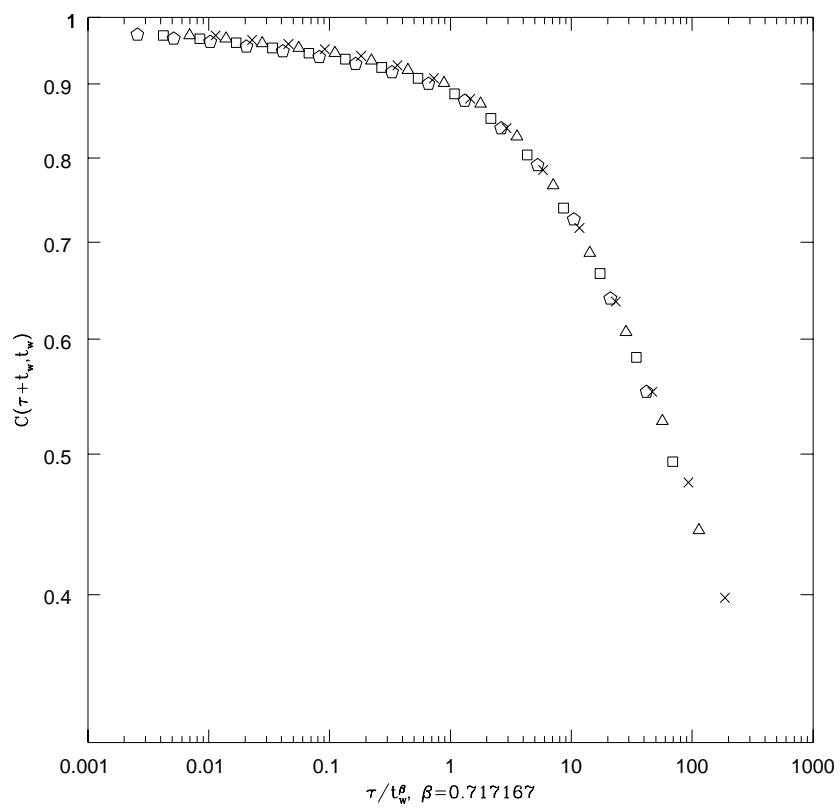

Figure 9: Scaling of the autocorrelation for $\epsilon=0.2$ and $t_{w}=2^{8}, 2^{10}, 2^{12}, 2^{14}$.

\section{Acknowledgments}

We acknowledge useful discussions with Juan Jesús Ruiz-Lorenzo.

\section{References}

[1] A. Crisanti and H. Sompolinsky, Phys. Rev. A 36 (1987) 4922; Phys. Rev. A 37 (1988) 4865.

[2] H. Sompolinsky, Phys. Rev. Lett. 47 (1981) 935; H. Sompolinsky and A. Zippelius, Phys. Rev. B 25 (1982) 6860.

[3] L. Cugliandolo and J. Kurchan, Phys. Rev. Lett. 71 (1993) 173; J. Phys. A (Math. Gen.) 27 (1994) 5749.

[4] M. Schreckenberg and H. Rieger, Z. Phys. B 86 (1992) 443.

[5] G. Iori and E. Marinari, J. Phys. A (Math. Gen.) 30 (1997) 4489.

[6] L. Cugliandolo, J. Kurchan, P. Le Doussal and L. Peliti, Phys. Rev. Lett. 78 (1997) 350. 
[7] E. Marinari, G. Parisi and J. J. Ruiz-Lorenzo, Numerical Simulations of Spin Glass Systems, in Spin Glasses and Random Field Systems, edited by A. P. Young (World Scientific, Singapore, 1997), 130.

[8] E. Marinari, G. Parisi and D. Rossetti, cond-mat/9708025.

[9] G. Parisi, F. Ricci-Tersenghi and J. J. Ruiz-Lorenzo, J. Phys. A (Math. Gen.) 29 (1996) 7943.

[10] H. Rieger, B. Steckemetz and M. Schreckenberg, Europhys. Lett. 27 (1994) 485. 